\title{
pH-Driven Reversible Assembly and Disassembly of Colloidal Gold Nanoparticles
}

\author{
Yun Liu ${ }^{1 * t}$, Weihua $\mathrm{Fu}^{2+}$, Zhongsheng $\mathrm{Xu}^{1}$, Liang Zhang ${ }^{1}$, Tao Sun ${ }^{1}$, Mengmeng $\mathrm{Du}^{1}$, \\ Xun Kang ${ }^{1}$, Shilin Xiao ${ }^{1}$, Chunyu Zhou ${ }^{1}$, Mingfu Gong ${ }^{1 *}$ and Dong Zhang ${ }^{1 *}$ \\ ${ }^{1}$ Department of Radiology, Xinqiao Hospital, Army Medical University, Chongqing, China, ${ }^{2}$ Department of Urology, Xinqiao \\ Hospital, Army Medical University, Chongqing, China
}

OPEN ACCESS

Edited by:

Zhiwei Li,

University of California, Riverside,

United States

Reviewed by:

Qinqin Liu,

Jiangsu University, China

Aiqin Gao,

Donghua University, China

*Correspondence:

Yun Liu

yunliu@tmmu.edu.cn

Mingfu Gong

hummer198625@163.com

Dong Zhang

hszhangd@tmmu.edu.cn

tThese authors have contributed equally to this work

Specialty section:

This article was submitted to Nanoscience,

a section of the journal

Frontiers in Chemistry

Received: 03 March 2021 Accepted: 09 March 2021

Published: 29 April 2021

Citation:

LiU Y, Fu W, Xu Z, Zhang L, Sun T,

Du M, Kang X, Xiao S, Zhou C,

Gong $M$ and Zhang $D$ (2021)

pH-Driven Reversible Assembly and

Disassembly of Colloidal Gold

Nanoparticles.

Front. Chem. 9:675491.

doi: 10.3389/fchem.2021.675491
Owing to the localized surface plasmon resonance (LSPR), dynamic manipulation of optical properties through the structure evolution of plasmonic nanoparticles has been intensively studied for practical applications. This paper describes a novel method for direct reversible self-assembly and dis-assembly of Au nanoparticles (AuNPs) in water driven by $\mathrm{pH}$ stimuli. Using 3-aminopropyltriethoxysilane (APTES) as the capping ligand and $\mathrm{pH}$-responsive agent, the APTES hydrolyzes rapidly in response to acid and then condenses into silicon. On the contrary, the condensed silicon can be broken down into silicate by base, which subsequently deprotonates the APTES on AuNPs. By controlling condensation and decomposition of APTES, the plasmonic coupling among adjacent AuNPs could be reversible tuned to display the plasmonic color switching. This study provides a facile and distinctive strategy to regulate the reversible self-assembly of AuNPs, and it also offers a new avenue for other plasmonic nanoparticles to adjust plasmonic properties via reversible self-assembly.

Keywords: gold nanoparticles, reversible self-assembly, pH-responsive, plasmonic, 3-aminopropyltriethoxysilane

\section{INTRODUCTION}

Noble metal nanoparticles have been intensively studied for a wide range of applications because of the localized surface plasmon resonance (LSPR), which is strongly dependent on nanoparticle size, shape, and composition (Gao et al., 2014; Chen et al., 2016). Low-dimensional plasmonic nanoparticle assemblies with new optical properties have recently attracted considerable attention because of the near-field coupling between adjacent particles (Liu D. et al., 2018; Li and Yin, 2019; Li et al., 2020a). The ideal way is the reversible assembly of such plasmonic nanostructures, which could enable dynamic tuning of the surface plasmon coupling by responding the external stimuli, and therefore by taking advantage of the ultrasensitive gap-dependent properties of plasmonic coupling, they have great promises for applications such as colorimetric sensors, bio- and chemical detection, and therapeutics (Bonacchi et al., 2016; Pillai et al., 2016; Liu L. et al., 2018; Zhou et al., 2021). Recent studies have demonstrated the reversible assembly by controlling the nanoparticle separation via various methods, for example, by modulating solvent composition to change the ionic strength of the solution, adding moisture, thermo-, photo-, magnetical-, and pH-responsive ligands, or reversible linking molecules (such as DNA) (Liu et al., 2012; Liu L. et al., 2019; Ding et al., 2016; Fan et al., 2016; He et al., 2016, 2019; Grzelczak et al., 2019; Li et al., 2020b; Severoni et al., 2020). However, these methods mentioned above more or less remain shortages, for example, the solutions usually contain two or more solvents, the responsive ligands (thiol molecules, biological molecules, or polymers) 
usually need complicated organic synthesis, and the reversible assembly process is not sensitive and robust. It is therefore highly worthy to design simple and effective strategies toward reversible assembly and dynamic tuning of the plasmonic coupling properties of noble metal nanoparticles.

Herein, for the first time, we develop a novel method for direct reversible assembly of $\mathrm{Au}$ nanoparticles (AuNPs) in water driven by $\mathrm{pH}$ stimuli with robust dynamic tuning of the surface plasmonic coupling among AuNPs. By using 3aminopropyltriethoxysilane (APTES) as the capping ligand and $\mathrm{pH}$-responsive agent, the APTES hydrolyzes rapidly in response to acid and then condenses into silicon. On the contrary, the condensed silicon can be broken down into silicate by base, which subsequently deprotonate the APTES on AuNPs. By controlling condensation and decomposition of APTES, the plasmonic coupling among adjacent AuNPs could be reversible tuned to display the plasmonic color switching. This study provides a facile and distinctive strategy to regulate the reversible self-assembly of AuNPs, and it also offers a new avenue for other plasmonic nanoparticles to adjust plasmonic properties via reversible self-assembly.

\section{MATERIALS AND METHODS}

\section{Materials}

Hydrogen tetrachloroaurate (III) trihydrate $\left(\mathrm{HAuCl}_{4}^{-} \cdot 3 \mathrm{H}_{2} \mathrm{O}\right)$ was purchased from Acros Organics, trisodium citrate $\left(\mathrm{Na}_{3} \mathrm{C}_{6} \mathrm{H}_{5} \mathrm{O}_{7} \cdot 2 \mathrm{H}_{2} \mathrm{O}\right)$ and sodium hydroxide $(\mathrm{NaOH})$ were obtained from Sino-pharm, ethanol (99.7\%) from Adamasbeta. 3-Aminopropyltriethoxysilane (APTES) used here was purchased from Fisher Scientific. All chemicals were analytic grade and used without further purification. All solutions were prepared in deionized water (DI water, $18.2 \mathrm{M} \Omega \cdot \mathrm{cm}$ ) from a Thermo Scientific Nanopure water purification system.

\section{Synthesis of Gold Nanoparticles}

Colloidal AuNPs with an average diameter of $15 \mathrm{~nm}$ were synthesized by the classical sodium citrate-reduction method proposed by Turkevich et al. (1951). Typically, $20 \mu \mathrm{l}$ of $2.5 \mathrm{~mol} / \mathrm{L}$ chloroauric acid solution was added to boiling DI water $(95 \mathrm{ml})$ with stirring, followed by the addition of an aqueous solution of trisodium citrate $(5 \mathrm{ml}, 1 \mathrm{wt} \%)$. The reaction was refluxed for $15 \mathrm{~min}$, and the solution gradually turned from colorless to wine red. The AuNPs were isolated by centrifugation, washed with DI water two times, and dispersed in $10 \mathrm{ml}$ of DI water. The size of the AuNPs was measured by transmission electron microscopy (TEM) analysis, and they were stored at $4^{\circ} \mathrm{C}$ in a glass vial for future use.

\section{Synthesis of Gold Nanoparticle Assemblies}

In a typical process, $0.1 \mathrm{ml}$ of AuNP solution was dispersed in $0.9 \mathrm{ml}$ of $\mathrm{DI}$ water, followed by the addition of an aqueous solution of APTES $\left(5 \mu \mathrm{l}, \mathrm{V}_{\mathrm{APTES}}: \mathrm{V}_{\mathrm{EtOH}}=1: 10\right)$. The assembly process could be clearly observed by the color change in the solution from wine red to bluish purple.

\section{Disassembly of Gold Nanoparticle Assemblies}

Typically, $10 \mu \mathrm{l}$ of $\mathrm{NaOH}$ solution $(0.5 \mathrm{M})$ was added into the dispersion of AuNP assemblies under manual shaking. A UVvis spectrometer (HR2000+CG-UV-NIR, Ocean Optics) was used to measure the real-time spectra changing during the disassembled process.

\section{Assembly of Gold Nanoparticles}

$\mathrm{HCl}$ solution $(10 \mu \mathrm{l})(0.5 \mathrm{M})$ was added into the disassembled dispersion of AuNP assemblies under manual shaking. The UVvis spectrometer (HR2000+CG-UV-NIR, Ocean Optics) was also used to record the changing of the real-time spectra during the re-assembled process.

\section{Characterization}

The optical properties of AuNPs and AuNP assemblies were measured by a UV-Vis spectrophotometer (HR2000+CG-UVNIR, Ocean Optics). The morphology of AuNP assemblies was performed on a transmission electron microscope (TEM, Hitachi HT7700) operated at $100 \mathrm{kV}$. Fourier transform infrared (FTIR) spectra were recorded on a Bruker ALPHA II spectrophotometer scanning from 4,000 to $400 \mathrm{~cm}^{-1}$ with a resolution of $4 \mathrm{~cm}^{-1}$. Dynamic light scattering (DLS) measurements were recorded on a zeta-potential and particle size analyzer (Z3000, Nicomp Particle Sizing Systems).

\section{RESULTS AND DISCUSSION}

The citrate-capped AuNPs with a diameter of $15 \mathrm{~nm}$ were synthesized by using the classic citrate reduction method and used in this paper for reversible assembly (Figure 1A). Once APTES was added into the Au solution, the amino group of APTES bonded with the AuNPs; dispersive AuNPs then proceeded into self-assembly owing to the hydrolysis of APTES, where the color of the solution changed from wine red to blue accompanied by a shoulder peak appearing at a longer wavelength around $650 \mathrm{~nm}$ (Supplementary Figure 1). APTES on the AuNP surface can be dynamically manipulated by $\mathrm{pH}$ variation, thereby enabling reversible assembly of AuNPs. The optical behavior of reversible assembly was also monitored as a function of $\mathrm{pH}$ using a UV-vis spectrometer. It was clear to see that upon increasing $\mathrm{pH}$ from 9.8 to 12.0 , the longer wavelength peak was blue shifted and eventually went back to the intrinsic peak of AuNPs at $520 \mathrm{~nm}$ (Figure 1B), with the color change in solution from blue, bluish purple, purple to wine red (Figure 1D), while the optical properties of AuNP assemblies present an inverse phenomenon with the $\mathrm{pH}$ decreasing from 12.0 to 9.8 (Figure 1C), accompanied by a solution color change from wine red, purple, bluish purple to blue (Figure 1D).

The morphologies of the reversible assembly of AuNPs were characterized by transmission electron microscope (TEM). Figure 2a shows that the isolated spherical AuNPs with an average size of $\sim 15 \mathrm{~nm}$ were successfully formed by using the classic citrate reduction method; then, the AuNPs gradually assembled into aggregates with increasing sizes after the addition of APTES (Figure 2b), which agreed with the UV-vis spectra data 

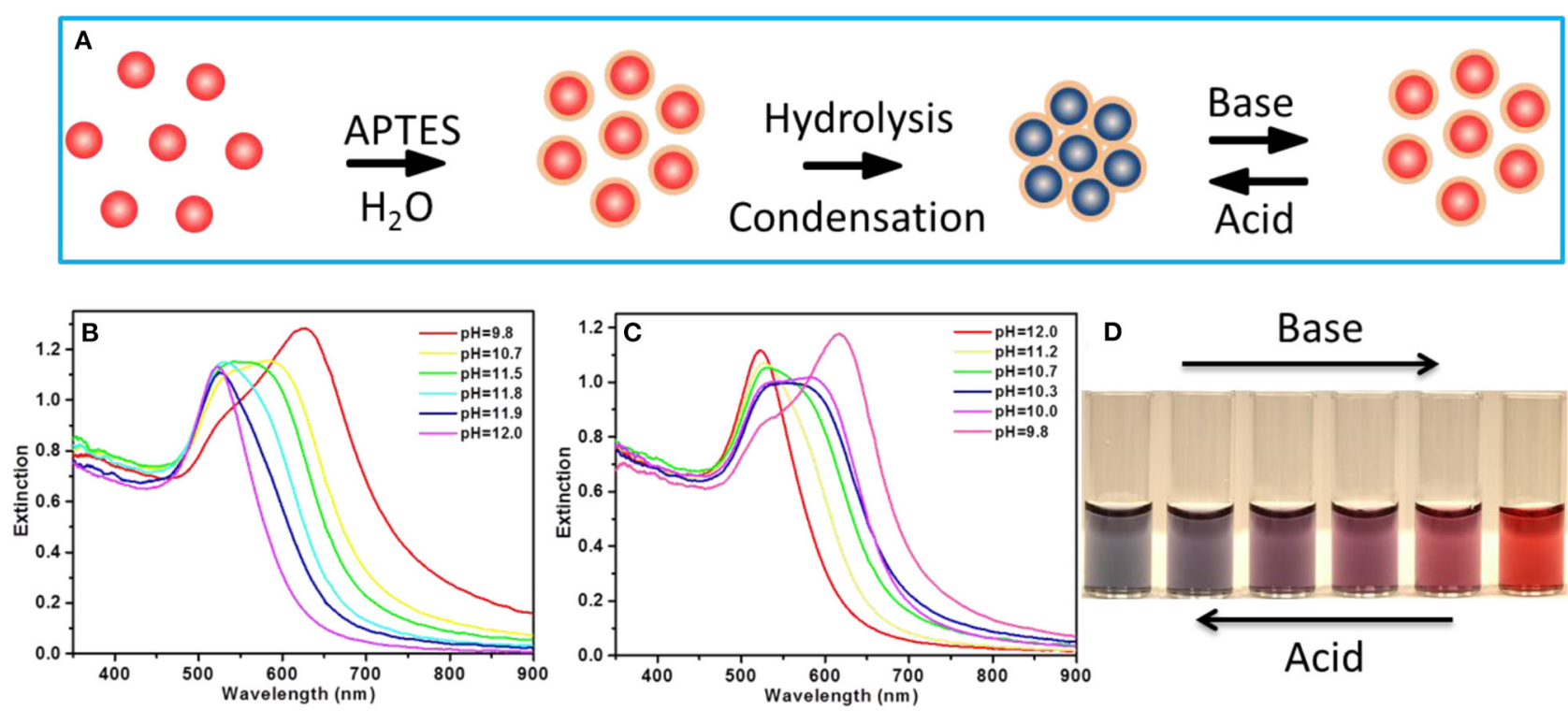

FIGURE 1 | (A) Schematic illustration of the reversible assembly of Au nanoparticles (AuNPs). (B) The UV-vis extinction spectra of the AuNP assemblies with pH increasing from 9.8 to 12.0. (C) The UV-vis extinction spectra of the AuNP assemblies with pH decreasing from 12.0 to 9.8. (D) The digital photo of the AuNP solution showing color evolution with $\mathrm{pH}$ variation.
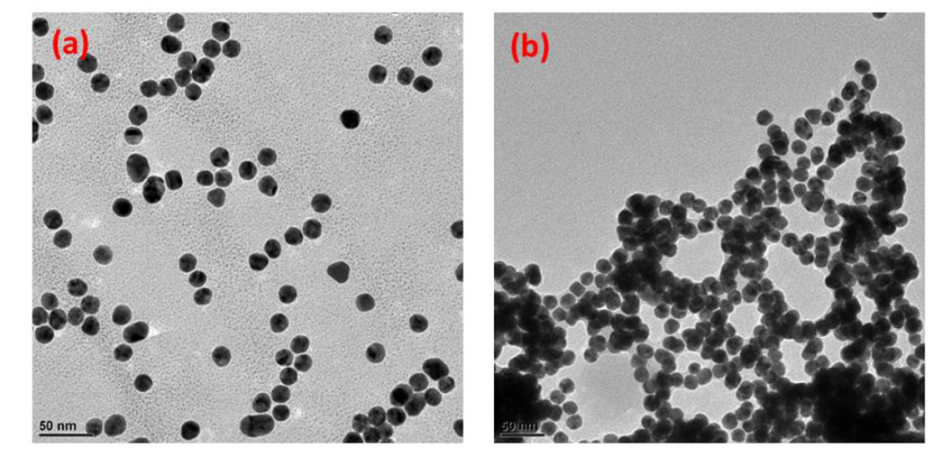

(e)
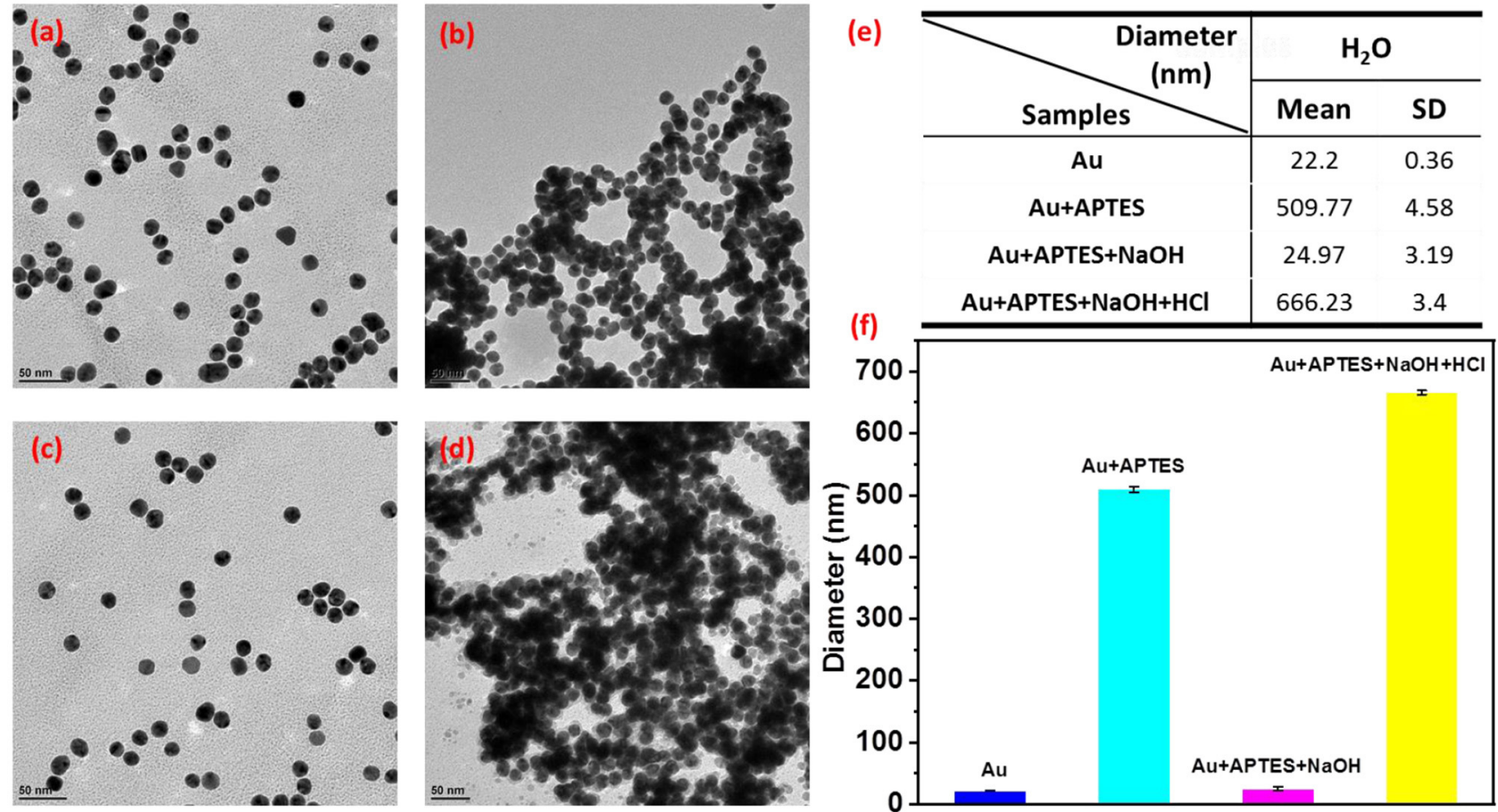

FIGURE 2 | Transmission electron microscopy (TEM) images of monodisperse AuNPs (a), the dispersion of AuNPs by adding 3-aminopropyltriethoxysilane (APTES) (b). The disassembly of AuNP assemblies with pH increasing from 9.8 to 12.0 (c), and the re-assembly of AuNPs with pH decreasing from 12.0 to 9.8 (d). (e,f) The size of AuNP assemblies and discrete AuNPs by dynamic light scattering (DLS).

(Supplementary Figure 1). In the reversible assembly process, the AuNP assemblies would be disassembled into smaller aggregates, eventually forming discrete nanoparticles with an increase in the solution $\mathrm{pH}$ to 12.0 (Figure 2c), while the nanoparticles could be re-assembled with a size increase by decreasing the $\mathrm{pH}$ to 9.8 (Figure $2 \mathbf{d}$ ). The disassembly and 

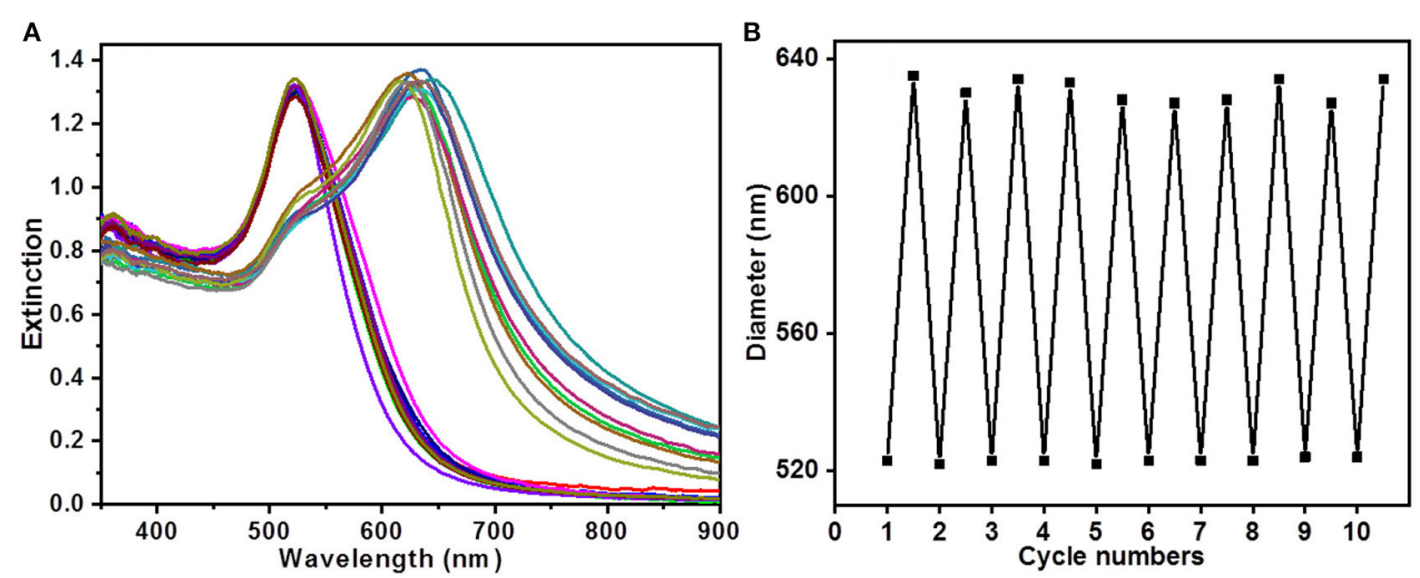

FIGURE 3 | (A) UV-vis spectra of 10 cycles switching between disassembled and assembled states. (B) Repeated changes of plasmonic peak positions in the 10 switching cycles.

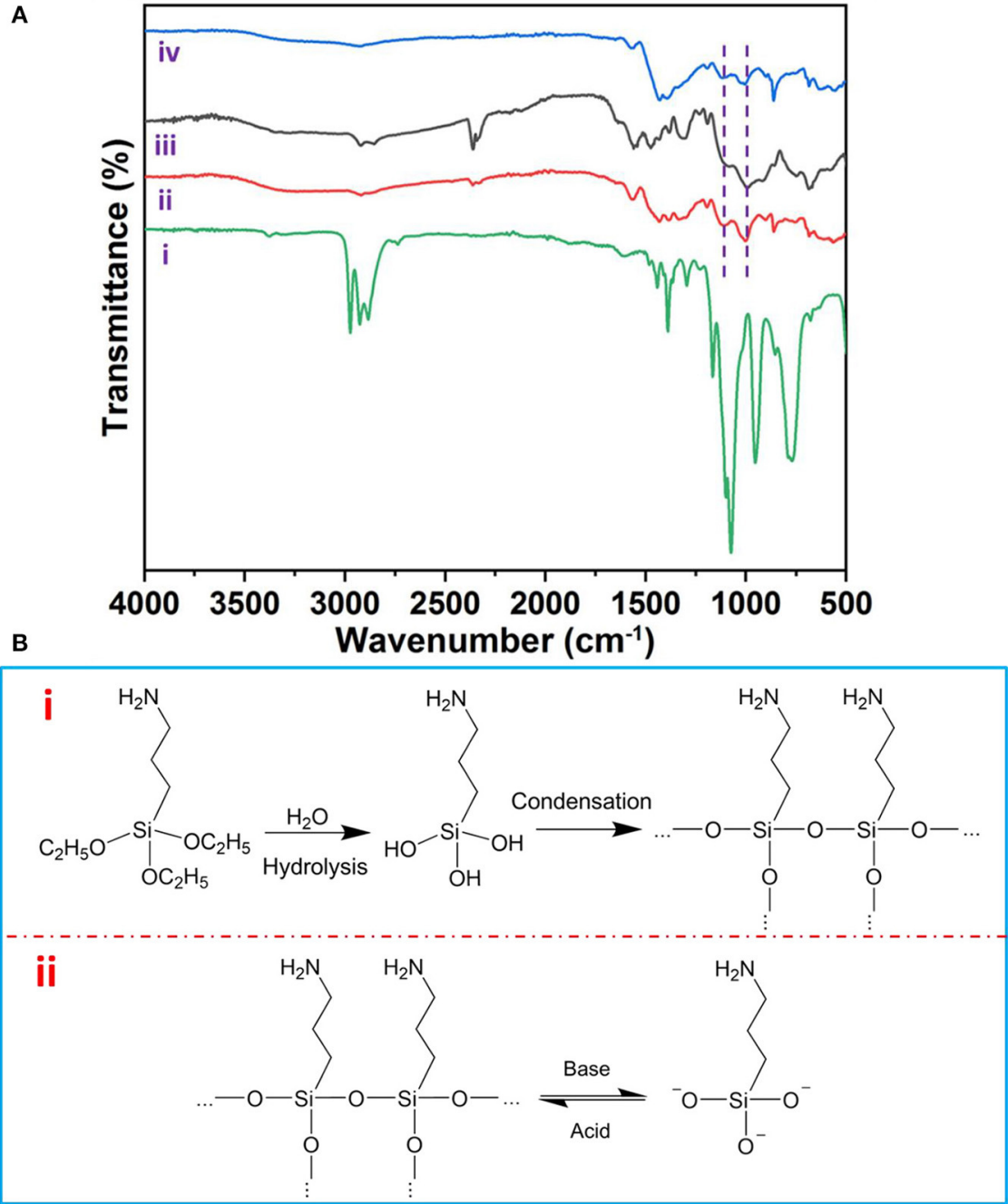

FIGURE 4 | (A) Fourier transform infrared (FTIR) spectra of APTES (i), initial (ii), disassembled (iii), and re-assembled AuNP assemblies (iv). (B) The mechanism of $\mathrm{pH}$-responsive reversible assembly of AuNPs. 
re-assembly behavior was further inspected by the dynamic light scattering (DLS) measurements conducted by adding different volumes of $\mathrm{NaOH}$ and $\mathrm{HCl}$ solutions into the solution of AuNP assemblies (Figures 2e,f). The average size of the AuNP assemblies decreased from about $509.77 \pm 4.58$ to $24.97 \pm$ $3.19 \mathrm{~nm}$ during disassembly, while the average size increased from $24.97 \pm 3.19$ to $666.23 \pm 3.4 \mathrm{~nm}$ during re-assembly, which agreed well with the TEM.

To investigate the stability and reversibility of the AuNP assemblies, the system was cycled 10 times by alternately adding $\mathrm{NaOH}$ and $\mathrm{HCl}$ into solution. As shown in the UV-vis spectra in Figure 3A, two absorption spectra corresponding to the extinction profiles could be switched by adding $\mathrm{NaOH}$ and $\mathrm{HCl}$ into the dispersion. Figure 3B plotted the peak positions of the coupled surface plasmon of the dispersion with the addition of $\mathrm{NaOH}$ and $\mathrm{HCl}$, respectively, which demonstrated impressive reversibility and reproducibility of the AuNP assemblies.

As shown in Figure 4A, Fourier transform infrared spectra (FTIR) detailedly revealed the asymmetric stretching modes of $\mathrm{Si}-\mathrm{O}-\mathrm{Si}$ bond peaking at 1,130 and $1,044 \mathrm{~cm}^{-1}$ (Majoul et al., 2015) on the surface of AuNPs, which could reversibly disappear and reappear upon the addition of $\mathrm{NaOH}$ and $\mathrm{HCl}$ solution, respectively. Therefore, we can draw the reversible assembly mechanism as follows: (i) APTES acts as the capping ligand bonded with AuNPs via the amino group of APTES (Feng et al., 2019). (ii) the APTES on the AuNPs' surface plays a key role as a $\mathrm{pH}$-responsive agent; the APTES itself hydrolyzes rapidly in response to acid and then condenses into silicon (De et al., 2000). On the contrary, the condensed silicon can be broken down into silicate by base (Zhang et al., 2008), which subsequently deprotonate the APTES on AuNPs (Figure 4B).

\section{CONCLUSIONS}

In summary, we have developed a novel method for direct reversible assembly of AuNPs in water driven by $\mathrm{pH}$ stimuli with robust dynamic tuning of the surface plasmonic coupling among AuNPs. APTES plays a critical role in the system, as

\section{REFERENCES}

Bonacchi, S., Cantelli, A., Battistelli, G., Guidetti, G., Calvaresi, M., Manzi, J., et al. (2016). Photoswitchable NIR-emitting gold nanoparticles. Angew. Chem. Int. Ed. 55, 11064-11068. doi: 10.1002/anie.201604290

Chen, P. C., Liu, X., Hedrick, J. L., Xie, Z., Wang, S., Lin, Q. Y., et al. (2016). Polyelemental nanoparticle libraries. Science 352, 1565-1569. doi: 10.1126/science.aaf8402

De, G., Karmakar, B., and Ganguli, D. (2000). Hydrolysis-condensation reactions of TEOS in the presence of acetic acid leading to the generation of glasslike silica microspheres in solution at room temperature. J. Mater. Chem. 10, 2289-2293. doi: 10.1039/b003221m

Ding, T., Rudrum, A. W., Herrmann, L. O., Turek, V., and Baumberg, J. J. (2016). Polymer-assisted self-assembly of gold nanoparticle monolayers and their dynamical switching. Nanoscale 8, 15864-15869. doi: 10.1039/C6NR 05199E

Fan, C., Bian, T., Shang, L., Shi, R., Wu, L. Z., Tung, C. H., et al. (2016). pH-Responsive reversible self-assembly of gold nanoparticles the capping ligand bonded with AuNPs via the amino group of APTES. Moreover, the APTES on the AuNPs' surface endows highly reversible assembly and dynamic color change to the system as the $\mathrm{Si}-\mathrm{O}-\mathrm{Si}$ bonds can be reversibly manipulated by controlling $\mathrm{pH}$ variation. Compared with the previous Au-based system, our system provides a facile and distinctive strategy exhibiting significant advantages such as higher color contrast, simpler procedure, better reversibility and reproducibility, and lower cost. In addition, this study also offers a new avenue for other plasmonic nanoparticles to adjust plasmonic properties via reversible assembly.

\section{DATA AVAILABILITY STATEMENT}

The original contributions presented in the study are included in the article/Supplementary Material, further inquiries can be directed to the corresponding author.

\section{AUTHOR CONTRIBUTIONS}

YL and WF designed and conducted some of the experiments, and wrote this paper. ZX, LZ, TS, and MD did the main experiment works. XK and SX processed some of the data. $\mathrm{CZ}$ processed the figures. MG and DZ reviewed and improved the paper.

\section{FUNDING}

The work was supported by the National Science Foundation of China (81903561), the Natural Science Foundation of Chongqing (cstc2020jcyj-msxmX1017), and the Army Medical University Foundation for Introducing Talents (YL).

\section{SUPPLEMENTARY MATERIAL}

The Supplementary Material for this article can be found online at: https://www.frontiersin.org/articles/10.3389/fchem. 2021.675491/full\#supplementary-material

into nanovesicles. Nanoscale 8, 3923-3925. doi: 10.1039/C6NR0 0044D

Feng, J., Yang, F., Wang, X., Lyu, F., Li, Z., and Yin, Y. (2019). Selfaligned anisotropic plasmonic nanostructures. Adv. Mater. 31:1900789. doi: 10.1002/adma.201900789

Gao, C., Hu, Y., Wang, M., Chi, M., and Yin, Y. (2014). Fully alloyed Ag/Au nanospheres: combining the plasmonic property of Ag with the stability of Au. J. Am. Chem. Soc. 136, 7474-7479. doi: 10.1021/ja502890c

Grzelczak, M., Liz-Marzán, L. M., and Klajn, R. (2019). Stimuli-responsive self-assembly of nanoparticles. Chem. Soc. Rev. 48, 1342-1361. doi: 10.1039/C8CS00787J

He, H., Feng, M., Chen, Q., Zhang, X., and Zhan, H. (2016). Light-induced reversible self-assembly of gold nanoparticles surface-immobilized with coumarin ligands. Angew. Chem. 128, 948-952. doi: 10.1002/ange.201508355

He, M. Q., Chen, S., Yao, K., Wang, K., Yu, Y. L., and Wang, J. H. (2019). Oriented assembly of gold nanoparticles with freezing-driven surface DNA manipulation and its application in SERS-based MicroRNA assay. Small Methods 3:1900017. doi: 10.1002/smtd.201900017 
Li, Z., Fan, Q., Wu, C., Li, Y., Cheng, C., and Yin, Y. (2020a). Magnetically tunable plasmon coupling of au nanoshells enabled by space-free confined growth. Nano Lett. 20, 8242-8249. doi: 10.1021/acs.nanolett.0c03350

Li, Z., Wang, W., and Yin, Y. (2020b). Colloidal assembly and active tuning of coupled plasmonic nanospheres. Trends Chem. 2, 593-608. doi: 10.1016/j.trechm.2020.03.008

Li, Z., and Yin, Y. (2019). Stimuli-responsive optical nanomaterials. Adv. Mater. 31:1807061. doi: 10.1002/adma.201807061

Liu, D., Fang, L., Zhou, F., Li, H., Zhang, T., Li, C., et al. (2018). Ultrasensitive and stable Au dimer-based colorimetric sensors using the dynamically tunable gap-dependent plasmonic coupling optical properties. Adv. Funct. Mater. 28:1707392. doi: 10.1002/adfm.201707392

Liu, L., Aleisa, R., Zhang, Y., Feng, J., Zheng, Y., Yin, Y., et al. (2019). Dynamic color-switching of plasmonic nanoparticle films. Angew. Chem. 131, 16453-16459. doi: 10.1002/ange.201910116

Liu, L., Gao, Z., Jiang, B., Bai, Y., Wang, W., and Yin, Y. (2018). Reversible assembly and dynamic plasmonic tuning of Ag nanoparticles enabled by limited ligand protection. Nano Lett. 18, 5312-5318. doi: 10.1021/acs.nanolett. $8 \mathrm{~b} 02325$

Liu, Y., Han, X., He, L., and Yin, Y. (2012). Thermoresponsive assembly of charged gold nanoparticles and their reversible tuning of plasmon coupling. Angew. Chem. Int. Ed. 51, 6373-6377. doi: 10.1002/anie.201201816

Majoul, N., Aouida, S., and Bessaïs, B. (2015). Progress of porous silicon APTES-functionalization by FTIR investigations. Appl. Surf. Sci. 331, 388-391. doi: 10.1016/j.apsusc.2015.01.107

Pillai, P. P., Kowalczyk, B., Kandere-Grzybowska, K., Borkowska, M., and Grzybowski, B. A. (2016). Engineering gram selectivity of mixed-charge gold nanoparticles by tuning the balance of surface charges. Angew. Chem. Int. Ed. 55, 8610-8614. doi: 10.1002/anie.201602965

Severoni, E., Maniappan, S., Liz-Marzán, L. M., Kumar, J., García de Abajo, F. J., and Galantini, L. (2020). Plasmon-Enhanced optical chirality through hotspot formation in surfactant-directed self-assembly of gold nanorods. ACS Nano 14, 16712-16722. doi: 10.1021/acsnano.0c03997

Turkevich, J., Stevenson, P. C., and Hillier, J. (1951). A study of the nucleation and growth processes in the synthesis of colloidal gold. Discuss. Faraday Soc. 11, 55-75. doi: 10.1039/df9511100055

Zhang, Q., Zhang, T., Ge, J., and Yin, Y. (2008). Permeable silica shell through surface-protected etching. Nano Lett. 8, 2867-2862. doi: 10.1021/nl8016187

Zhou, C., Zhang, L., Sun, T., Zhang, Y., Liu, Y., Gong, M., et al. (2021). Activatable NIR-II plasmonic nanotheranostics for efficient photoacoustic imaging and photothermal cancer therapy. Adv. Mater. 33:2006532. doi: 10.1002/adma.202006532

Conflict of Interest: The authors declare that the research was conducted in the absence of any commercial or financial relationships that could be construed as a potential conflict of interest.

Copyright (C) 2021 Liu, Fu, Xu, Zhang, Sun, Du, Kang, Xiao, Zhou, Gong and Zhang. This is an open-access article distributed under the terms of the Creative Commons Attribution License (CC BY). The use, distribution or reproduction in other forums is permitted, provided the original author(s) and the copyright owner(s) are credited and that the original publication in this journal is cited, in accordance with accepted academic practice. No use, distribution or reproduction is permitted which does not comply with these terms. 